\title{
Septoplasty for nasal obstruction due to a deviated nasal septum in adults: a systematic review*
}

\author{
M.M.H.T. van Egmond', M.M. Rovers ${ }^{2,3}$, A.H.J. Tillema ${ }^{4}$, N. van Heerbeek' \\ 'Department of Otorhinolaryngology, \\ 2 Department of Operating Rooms, \\ ${ }^{3}$ Department of Health Evidence, \\ ${ }^{4}$ Radboud University Medical Library, \\ Radboud Institute for Health Sciences, Radboud university medical center Nijmegen, the Netherlands
}

Rhinology 56: 3, 195-208, 2018

https://doi.org/10.4193/Rhin18.016

*Received for publication:

January 25, 2018

Accepted: February 27, 2018

Background: The status of current evidence for the effectiveness of septoplasty is unclear. This systematic review evaluates the effectiveness of a) septoplasty (with or without concurrent turbinate surgery) versus non-surgical management, and b) septoplasty with concurrent turbinate surgery versus septoplasty alone, for nasal obstruction due to a deviated nasal septum in adults.

Methodology: Eligible for inclusion were randomised controlled trials and non-randomised designs comparing treatment strategies. Risk of bias was assessed using Cochrane's tool. Standardised mean differences and risk differences with $95 \%$ confidence intervals were calculated. Substantial heterogeneity between included studies did not allow meta-analyses.

Results: No studies were found comparing septoplasty (with or without concurrent turbinate surgery) to non-surgical management, but 11 articles were included to compare septoplasty with concurrent turbinate surgery to septoplasty alone. Five studies described both subjective and objective outcomes; six studies reported one or the other. Risk of bias was overall high. Although outcomes generally improved after treatment, eight out of nine studies on subjective measures and five out of seven studies on objective measures found no additional benefit of turbinate surgery.

Conclusions: Despite the routine application of septoplasty in clinical practice, the current body of evidence does not support firm conclusions on its effectiveness.

Key words: nasal obstruction, nasal septum, nasal surgical procedures, quality of life, turbinates

\section{Introduction}

Septoplasty, i.e., surgical correction of the deviated nasal septum, is the most common ENT-operation in adults ${ }^{(1)}$. However, indications seem practice-based rather than evidence-based and internationally accepted guidelines are lacking ${ }^{(2)}$. Annual septoplasty rates differ accordingly between countries. The number of septoplasties per 10,000 inhabitants was 3.9 in England, 6.6 in the Netherlands, and 12.2 in Germany in $2014^{(3-5)}$. In the United States, the annual septoplasty rate was 8.7 per 10,000 inhabitants in $2006^{(6)}$.

The main indication for septoplasty is nasal obstruction, commonly defined as an unpleasant sensation of insufficient airflow through the nose ${ }^{(7)}$. Nasal obstruction is associated with mucosal as well as anatomical conditions. Underlying patho- genesis may be multifactorial. The most frequent anatomical cause is a deviated nasal septum, which can be accompanied by hypertrophy of the turbinate contralateral to the deviation ${ }^{(8)}$. Septoplasty (with or without concurrent turbinate surgery) is performed to widen nasal passages and thereby improve nasal airflow ${ }^{(9)}$.

Nonetheless, the effectiveness of septoplasty and additional benefits of turbinate surgery are questioned. According to the literature, nasal septal deviation may have a prevalence of up to $80 \%$, whereas only a minority suffers from nasal obstruction. Whether straightening the deviated septum provides any benefit to those patients, remains a topic of debate in ENT-practice ${ }^{(10)}$. The American Academy of Otolaryngology - Head and Neck Surgery initiated a consensus panel on septal surgery, which 
failed to reach agreement in over one third of the 33 controversial clinical dilemmas discussed ${ }^{(11)}$. Both in the United Kingdom and in the Netherlands, professional associations of ENT-surgeons recognised a need for evidence to advance the debate on indications for and benefits of septoplasty ${ }^{(12,13)}$.

The lack of clinical consensus is, however, accompanied by scarcity of scientific literature. Randomised controlled trials seem underrepresented and the status of (other) existing evidence is unclear. Remarkably, this does not appear to hamper the routine application of septoplasty in daily practice ${ }^{(2)}$. Therefore we decided to perform a systematic review of available evidence, including non-randomised designs. The aims of this systematic review are: to evaluate the effectiveness of a) septoplasty (with or without concurrent turbinate surgery) versus non-surgical management, and b) septoplasty with concurrent turbinate surgery versus septoplasty alone, for nasal obstruction due to a deviated nasal septum in adults. By discussing the findings, strengths, and weaknesses of available studies, we intend to assess the status of current evidence for the effectiveness of septoplasty.

\section{Materials and methods}

Protocol registration

The review protocol can be accessed at the website of PROSPE$\mathrm{RO}$, the International Prospective Register of Systematic Reviews (https://www.crd.york.ac.uk/PROSPERO/). The protocol was registered under the number CRD42017060632 on March 31, 2017.

\section{Eligibility criteria}

Participants

Studies in adults with nasal obstruction due to a deviated nasal septum were considered eligible for inclusion in this review. In clinical practice, nasal obstruction due to a deviated nasal septum is primarily diagnosed by an internal exam of the nose, consisting of anterior rhinoscopy and nasal endoscopy. The internal exam is performed by the ENT-surgeon to assess whether the deviation causes a mechanical nasal airway obstruction, leading to impaired nasal breathing ${ }^{(14)}$. For this review, the study authors' definition of nasal obstruction due to a deviated nasal septum was adopted. In included studies, nasal obstruction had to be the primary indication for performing septoplasty. Studies in which patients were selected for septoplasty because of other complaints (e.g., impairment of normal sinus drainage, sleep disorders, headaches) were excluded. Studies in the following patient categories were also excluded: patients with a history of nasal septal surgery; patients with nasal septal perforation; patients with untreated allergic rhinitis or allergic rhinitis unresponsive to medical treatment; and cleft lip and/or palate patients.

\section{Intervention and comparison}

Included studies had to compare septoplasty (with or without concurrent turbinate surgery) to non-surgical management, or septoplasty with turbinate surgery to septoplasty alone. Nonsurgical management could consist of watchful waiting and medical treatment, such as local or systemic steroids and antihistamines. Studies in which septoplasty was combined with other procedures than turbinate surgery (e.g., rhinoplasty, spreader grafts, butterfly grafts, FESS, adenoidectomy) were excluded.

\section{Outcomes}

Follow-up needed to be at least three months to prevent direct postoperative effects like mucosal swelling from distorting outcome assessment. Desirable time points of outcome assessment were three months, six months, 12 months, and 24 months. Both subjective (e.g., health-related quality of life) as well as objective (e.g., nasal patency) outcome measures were taken into account. Health-related quality of life may be measured using patientbased questionnaires such as the Glasgow Benefit Inventory (GBI), Nasal Obstruction Symptom Evaluation (NOSE) Scale, and the Sino-Nasal Outcome Test (SNOT) ${ }^{(15-17)}$. Visual Analog Scales (VAS) or Likert scales can be applied to grade symptom severity ${ }^{(18,19)}$. For the objective assessment of nasal patency, several outcome measures are available, e.g., Peak Nasal Inspiratory Flow (PNIF), Acoustic Rhinometry (AR), or Active Anterior Rhinomanometry (AAR), which may be performed with a Four-Phase Rhinomanometer (4PR) ${ }^{(14,20,21)}$.

\section{Other eligibility criteria}

The preferred study design was a randomised controlled trial comparing either septoplasty to non-surgical management, or septoplasty with concurrent turbinate surgery to septoplasty alone. We were, however, apprehensive of not finding any RCTs. As it was our aim to assess the status of currently available evidence, we also considered the following study designs for inclusion in this review: quasi-randomised trials; cohort studies comparing interventions; non-randomised controlled trials; case control studies; and controlled before-and-after studies. We excluded opinion articles, animal studies, (systematic) reviews, case reports, conference abstracts, and studies on other interventions (e.g., nasal packing, various analgesia, postoperative care).

\section{Information sources and search strategy}

We systematically searched PubMed, the Cochrane Library (both from inception) and Ovid EMBASE (from 1974) up to October 10, 2017 for studies on septoplasty for nasal obstruction in adults with a deviated nasal septum. Terms relating to the patients, intervention, and outcomes were included in the search strategy, which combined synonyms for nasal obstruction, nasal septal deviation, septoplasty, turbinate surgery, and various subjective 
as well as objective outcome measures. Both keywords (MeSH and Emtree) and free-text terms in title and abstract were included in the search query. Intervention terms were combined with nose-related synonyms to minimise noise from cardiovascular studies on surgery of the interventricular septum. No language or date restrictions were applied. In addition to the electronic search, articles' reference lists were scanned for any applicable studies that had not yet been identified.

\section{Study selection}

The results of the search strategy were merged and duplicates were removed using EndNote reference management software (version X7, Thomas Reuters, New York City, NY, USA). Two review authors (MvE, $\mathrm{NvH}$ ) individually screened titles and abstracts to identify relevant reports based on the inclusion and exclusion criteria outlined above. Full texts of these potentially relevant studies were retrieved by a librarian (AT) and independently assessed for eligibility by two reviewers (MvE and $\mathrm{NvH}$ ). Any disagreements were resolved by discussion with a third reviewer (MR).

\section{Data extraction}

Data extraction was conducted by one reviewer (MvE) using a pre-defined form. Unclear issues were discussed with two other reviewers ( $\mathrm{NvH}, \mathrm{MR})$ and resolved by consensus. The following data were extracted from included studies: study design; description of participants (eligibility criteria, total number, mean age, gender, country of origin, type and severity of nasal septal deviation, prior treatment); total number of intervention groups; intervention details (type of surgery or specifics of non-surgical treatment); number of participants allocated to each intervention group; total duration of follow-up; time points of outcome assessment during follow-up; primary and secondary outcomes collected and reported; missing data for each intervention group; summary data for each intervention group; and the authors' conclusions.

\section{Risk of bias assessment}

Risk of bias in included studies was independently assessed by two review authors (MvE, NvH). Any differences in opinion were resolved by discussion with a third review author (MR). Included studies were evaluated using Cochrane's risk of bias tool, which comprises a critical assessment of random sequence generation, allocation concealment, blinding of participants and personnel, blinding of outcome assessment, incomplete outcome data, selective reporting, and other sources of bias ${ }^{(22)}$. Each domain in every individual study was assigned either a high, low, or unclear risk of bias, based on the study report and, if applicable, correspondence with study authors. Blinding of outcome assessment was scored separately for subjective and objective outcomes. Since non-randomised studies were also considered for in-
Figure 1. Flow diagram of study selection.

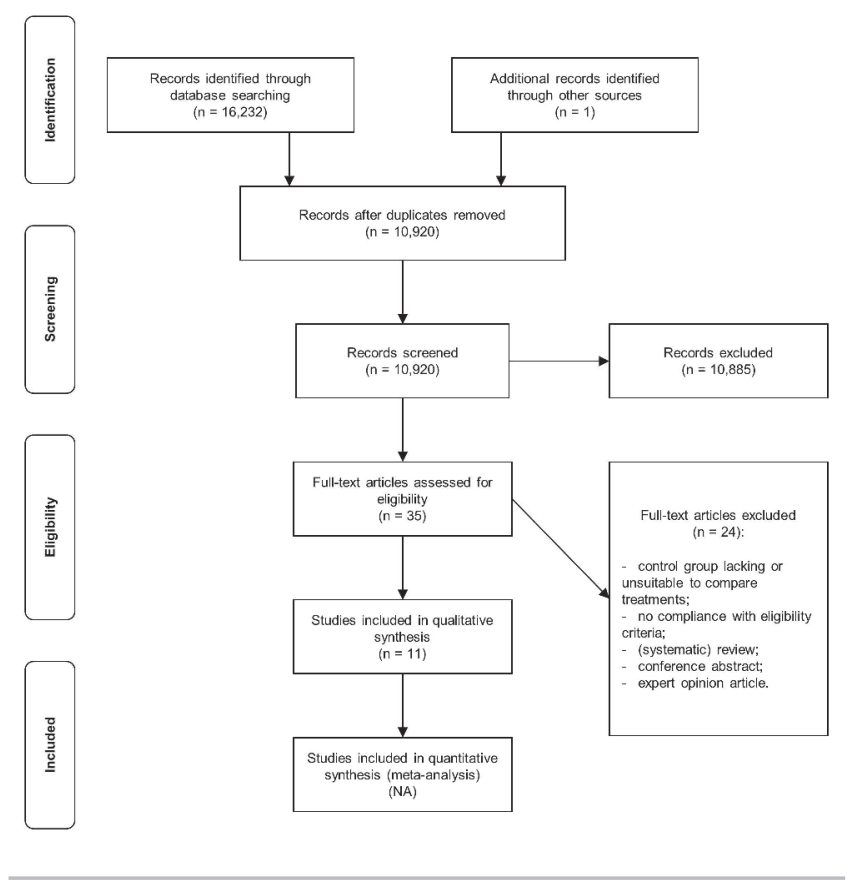

clusion in this review, we paid special attention to the execution of the studies and the risk of selection bias, confounding, and reporting bias including selective reporting of outcomes. For all included studies, it was evaluated whether a study protocol was available and if so, whether the study's pre-specified outcomes had been reported. Moreover, we intended to quantify publication bias with a funnel plot of the intervention effect estimate on the horizontal axis and the measure of study size on the vertical axis, but this proved to be impossible due to the great variety in outcome measures applied across a small number of included studies. Results of risk of bias assessment were graphically summarised using Review Manager 5 (RevMan5) software (version 5.3, Cochrane Collaboration, London, England).

\section{Summary measures and synthesis of results}

We planned to calculate standardised mean differences (for continuous outcomes) and risk differences (for dichotomous outcomes) with their corresponding $95 \%$ confidence intervals using RevMan 5 software. Ultimately, the studies included in our systematic review were too heterogeneous to perform meta-analyses, see also Table 1. For this reason, effect estimates reported in the individual studies were presented.

\section{Results}

\section{Study selection}

Our systematic search of PubMed, the Cochrane Library, and Ovid EMBASE yielded a total of 16,232 records, which was reduced to 10,919 results after removing duplicates. By scanning articles' reference lists we found one additional study, which was 
Table 1. Schematic overview of clinical and methodological differences between included studies.

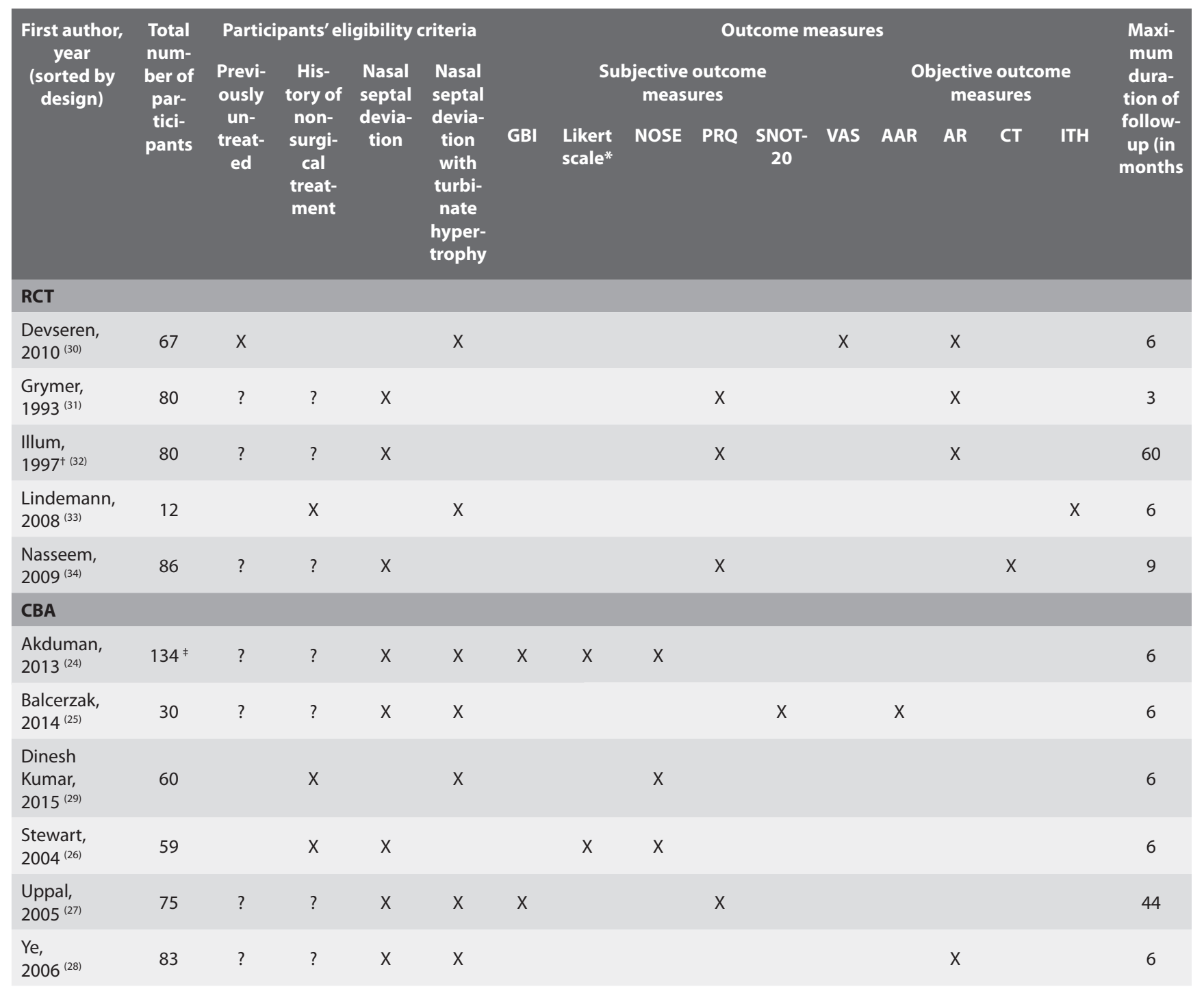

* Likert scale ranged 5 points in Akduman et al. and 11 points in Stewart et al. ${ }^{\dagger}$ Long-term follow-up of Grymer et al. ${ }^{\ddagger}$ The study authors included 35 patients who underwent septoplasty and additional valve surgery; effect estimates of these patients were not included in this systematic review. AAR: Active Anterior Rhinomanometry. AR: Acoustic Rhinometry. CBA: Controlled Before-and-After study. CT: Computed Tomography measurements of turbinate thickness. GBI: Glasgow Benefit Inventory. NOSE: Nasal Obstruction Symptom Evaluation scale. PRQ: Patient-Reported Questionnaire concerning nasal symptoms or treatment satisfaction; not otherwise specified by study authors. RCT: Randomised Controlled Trial. SNOT-20: Sino-Nasal Outcome Test-20. ITH: Inspiratory air Temperature and absolute Humidity. VAS: Visual Analogue Scale.

potentially applicable even though it had not been indexed in either one of the three databases searched. Based on title and abstract, 10,885 articles needed to be excluded due to incompatibility with our eligibility criteria. Full-texts of the remaining 35 studies were screened and another 24 articles were excluded for the following reasons: in 14 studies, the control group either lacked or was unsuitable to compare treatments; five studies did not comply with our eligibility criteria concerning patients, interventions, or follow-up; two articles were (systematic) reviews; two articles were conference abstracts; and one publication was solely based on expert opinion. A total of 11 articles could be included in this systematic review. A flow diagram of study selection is shown in Figure 1, derived from The Preferred Reporting Items for Systematic Reviews and Meta-Analyses (PRISMA) Group ${ }^{(23)}$.

\section{Study characteristics}

The first aim of this review was to assess the effectiveness of septoplasty (with or without concurrent turbinate surgery) versus non-surgical management for nasal obstruction due to 


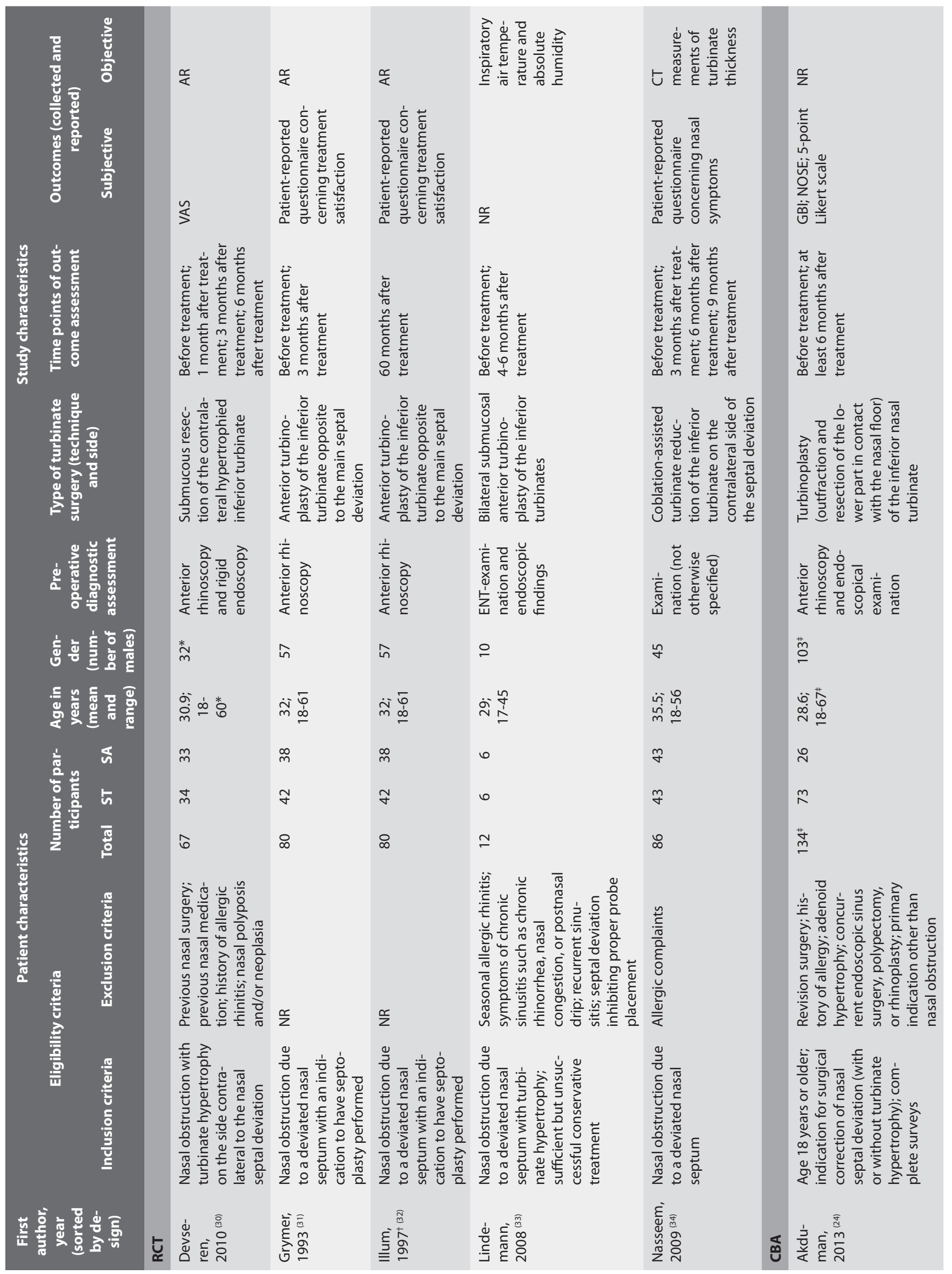




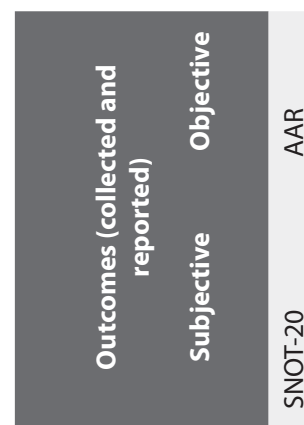

$\stackrel{\propto}{z}$

$\stackrel{\frac{c}{z}}{2}$
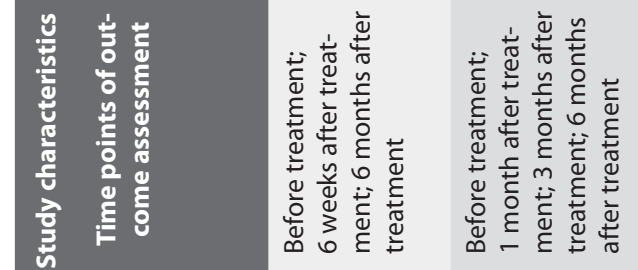

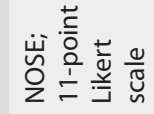

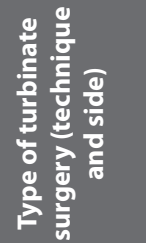

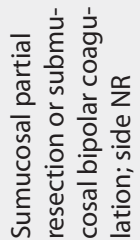

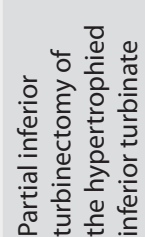

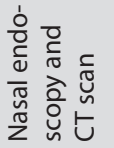

$\stackrel{\frac{m}{z}}{2}$

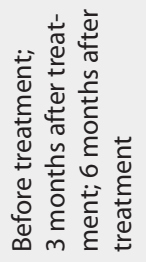

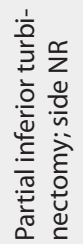

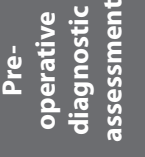

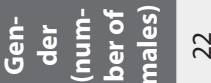

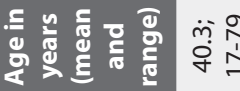

$\frac{\Re}{z}$

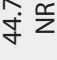

咅的的

웅

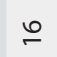

$\stackrel{m}{q}$

8

ㅇํ

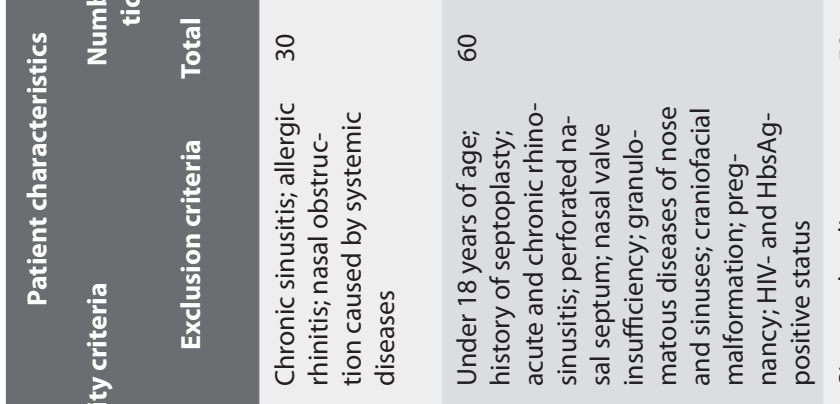

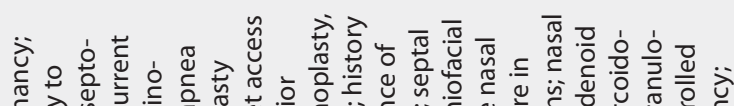

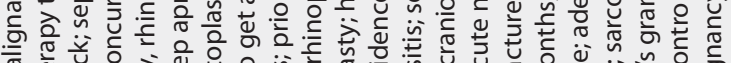

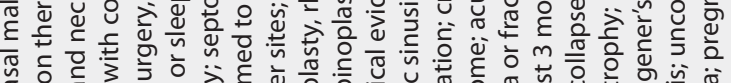

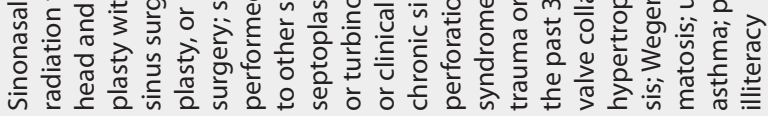

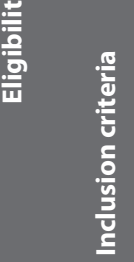

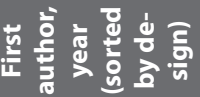

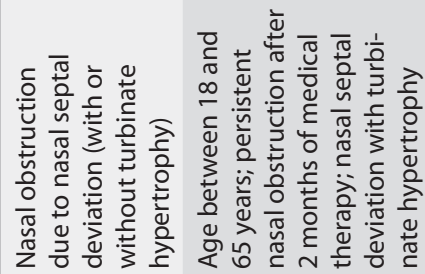

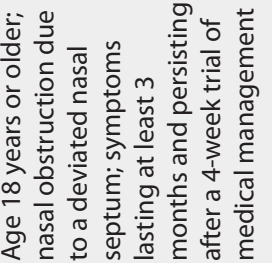

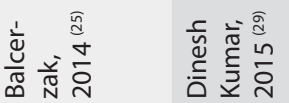

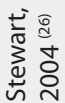




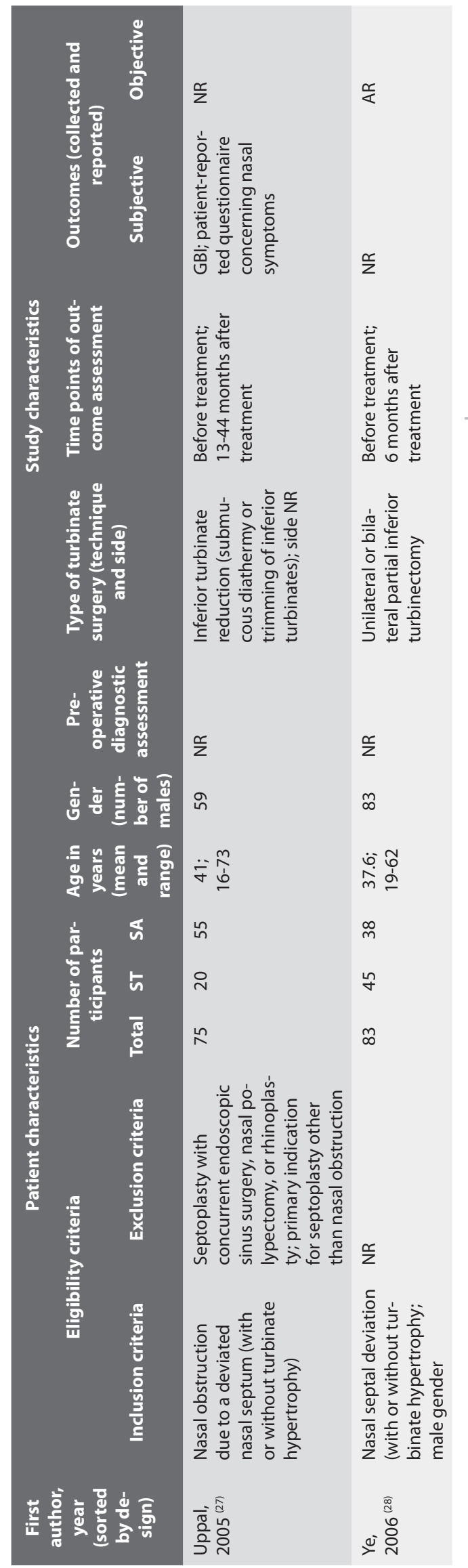

*The study authors have calculated mean age, age range, and number of males based on 42 patients, as 25 patients were lost to follow-up and excluded from further analyses. ${ }^{\dagger}$ Long-term follow-up of Grymer et al. ${ }^{\ddagger}$ The study authors included 35 patients who underwent septoplasty and additional valve surgery; effect estimates of these patients were not included in this systematic review. Mean age, age range, and number of males were presented based on the total sample of 134 patients. AAR: Active Anterior Rhinomanometry. AR: Acoustic Rhinometry. CBA: Controlled Before-and-After study. GBI: Glasgow Benefit Inventory. NOSE: Nasal Obstruction Symptom Evaluation scale. NR: Not Reported. RCT: Randomised Controlled Trial. SA: Septoplasty Alone. SNOT-20: SinoNasal Outcome Test-20. ST: Septoplasty with concurrent Turbinate surgery. VAS: Visual Analogue Scale.

a deviated nasal septum in adults. We were not able to include any studies with respect to this comparison.

The second aim was to compare septoplasty with concurrent turbinate surgery versus septoplasty alone for nasal obstruction due to a deviated nasal septum in adults. For this comparison, five randomised controlled trials and six controlled before-andafter studies (in which measurements were performed before and after treatment, both in patients undergoing septoplasty with concurrent turbinate surgery and in patients undergoing septoplasty alone) could be included. The number of included participants per study varied between 12 and 134 patients. The preoperative diagnostic assessment consisted of anterior rhinoscopy and nasal endoscopy in most cases. The type of turbinate surgery was often described as (anterior) turbinoplasty or partial turbinectomy. In the majority of studies, turbinate surgery was unilateral. Table 2 provides an overview of included studies and details on their methods, participants, interventions, and outcomes.

\section{Risk of bias assessment}

Results of risk of bias assessment are graphically summarised in Figures 2 and 3. In Figure 2, judgments about each risk of bias item are presented as percentages across all included studies, whereas Figure 3 shows scores on each risk of bias item for every included study separately.

\section{Sequence generation and allocation concealment} In five out of 11 included studies, the indication to have septoplasty performed with or without turbinate surgery was based on clinical judgment or patient preferences ${ }^{(24-28)}$. In one study, correspondence with study authors learned that patients were alternately divided between two groups ${ }^{(29)}$. The remaining five studies all mentioned a random component in the sequence generation process. None of these studies, however, reported an adequate method of allocation concealment ${ }^{(30-34)}$. 
Random sequence generation (selection bias)

Allocation concealment (selection bias)

Blinding of participants and personnel (performance bias)

Blinding of outcome assessment (detection bias): subjective outcomes

Blinding of outcome assessment (detection bias): objective outcomes

Incomplete outcome data (attrition bias)

Selective reporting (reporting bias)

Other bias

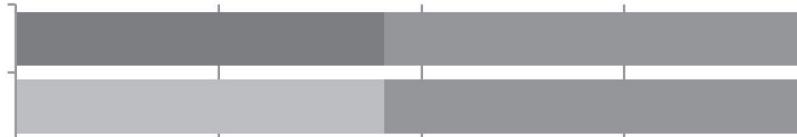

\section{政}

\section{五}

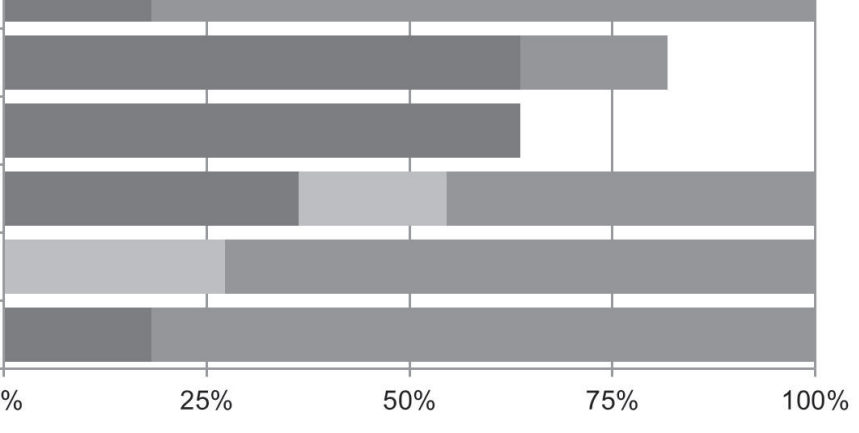

- Low risk of bias

- Unclear risk of bias

- High risk of bias

Figure 2. Risk of bias graph: review authors'judgment about each risk of bias item presented as percentages across all included studies.

\section{Blinding}

Only two publications described efforts to prevent performance bias due to knowledge of the allocated interventions. Both in Balcerzak et al. and Stewart et al., ENT-surgeons were not involved in collecting follow-up data ${ }^{(25,26)}$. Moreover, Stewart et al. blinded physicians to patients' pre- and postoperative scores on study outcomes ${ }^{(26)}$.

The risk of detection bias was assessed separately for subjective and objective outcomes. In all included studies, patients were aware of the type of surgery performed. Taking the patients' perspective into account, we estimated that the difference in perceived desirability between two types of surgical treatment would be less pronounced than between septoplasty and nonsurgical management. For this reason, most subjective outcome measurements were considered unlikely to be influenced by lack of blinding. The risk of detection bias was found to be high only in Grymer et al. and Illum et al., since subjective outcomes of these studies mainly addressed satisfaction with the treatment received ${ }^{(31,32)}$. For objective outcomes, the risk of detection bias was judged to be low irrespective of the lack of blinding.

\section{Incomplete outcome data and selective reporting}

Four studies reported that all outcome data were complete ${ }^{(27,}$ 28,33,34). In two studies, no information on missing outcome data was provided ${ }^{(25,29)}$. Four other studies presented proportions of missing outcomes, but reasons for loss to follow-up were rarely stated and adequate methods for handling incomplete outcome data were never described ${ }^{(26,30-32)}$. Some studies appeared to have adopted a per protocol approach by simply excluding dropouts ${ }^{(24,30,31)}$.

A study protocol could not be obtained for any of the included studies. Moreover, none of the randomised controlled trials were listed on ClinicalTrials.gov or in the ISRCTN (International
Standard Randomised Controlled Trial Number) Registry. Consequently, it was impossible to verify whether all of the studies' pre-specified outcomes had been published. Obvious evidence of selective outcome reporting was identified only in Stewart et al., where one of the outcome measures listed in the Methods section (i.e., an 11-point Likert scale) was entirely omitted from the Results ${ }^{(26)}$. Additionally, risk of reporting bias was high in seven other studies, whose summary measures could not be calculated due to incomplete reporting of outcomes (24, 27, 29-33).

\section{Other potential sources of bias}

Systematic differences in baseline characteristics between the two groups were likely to have occurred especially in studies allocating treatments based on clinical judgment or patient preferences ${ }^{(24-28)}$.

Furthermore, specific issues that raised concern about the possibility of bias were identified in two of the controlled before-andafter studies and three of the included RCTs. In Akduman et al., patients were allocated to septoplasty alone, septoplasty with concurrent turbinate surgery, or septoplasty with additional valve surgery ${ }^{(24)}$. Given this third treatment option, the selection of patients enrolled in our two groups of interest may have been different, had only two options been present. Dinesh Kumar et al. presented inconsistent tables, which showed different numbers for the same outcomes ${ }^{(29)}$. In Devseren et al. and Grymer et al., respectively two and six patients with postoperative complications were excluded from the analyses ${ }^{(30,31)}$. Nasseem et al. performed additional turbinate surgery in patients allocated to septoplasty alone in case of persistent complaints ${ }^{(34)}$.

\section{Study results}

A summary of findings from included studies is provided in Table 3. In case of repeated measurements, we planned to 


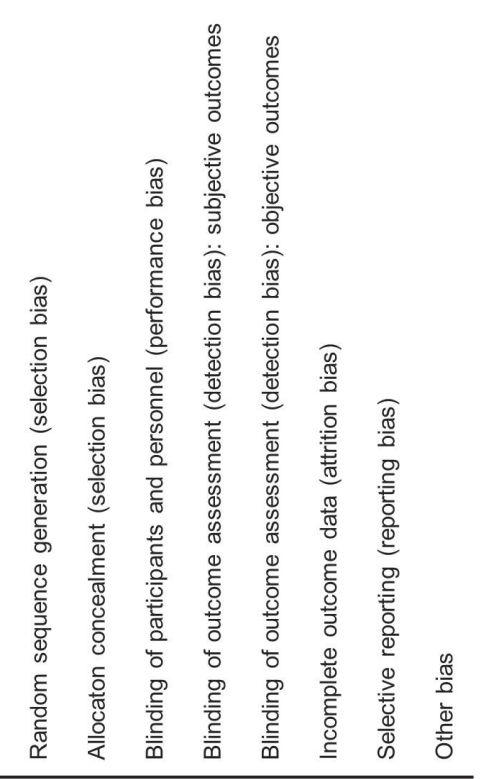

\begin{tabular}{llllllllll}
\hline Adkuman 2013 & - & - & - & + & & - & - & - \\
\hline Balcerzak 2014 & - & - & + & + & + & $?$ & $?$ & - \\
\hline Devseren 2010 & + & $?$ & - & + & + & - & - & - \\
\hline $\begin{array}{l}\text { Dinesh Kumar } \\
2015\end{array}$ & - & - & - & + & & $?$ & - & - \\
\hline Grymer 1993 & + & $?$ & - & - & + & - & - & - \\
\hline Illum 1997 & + & $?$ & - & - & + & - & - & + \\
\hline Lindemann 2008 & + & $?$ & - & & + & + & - & + \\
\hline Nasseem 2009 & + & $?$ & - & + & + & + & $?$ & - \\
\hline Stewart 2004 & - & - & + & + & & - & - & - \\
\hline Uppal 2005 & - & - & - & + & & + & - & - \\
\hline Ye 2006 & - & - & - & & + & + & $?$ & - \\
\hline
\end{tabular}

Figure 3. Risk of bias summary: review authors' judgment about each risk of bias item for every included study.

present outcomes at 12 months. In none of the included studies, however, data were collected at one year of follow-up. Median follow-up was six months; data at this time point were presented whenever possible ${ }^{(24-26,28-30,33,34)}$. In the remaining three studies, no repeated measurements were conducted. Therefore, we presented outcomes at the time point selected by study authors, which ranged between three and 60 months ${ }^{(27,31,32)}$.

\section{Subjective outcome measures}

Subjective outcomes were reported in nine out of 11 included studies ${ }^{(24-27,29-32,34)}$. Six different subjective outcome measures could be distinguished, some of which were applied in only one study (i.e., SNOT-20, VAS) and others in two to three studies (i.e., GBI, Likert scale, NOSE); four studies assessed nasal symptoms or treatment satisfaction using a patient-reported questionnaire (PRQ) which was not otherwise specified by study authors. Eight out of nine studies reported subjective benefit after treatment, irrespective of whether septoplasty had been performed with or without concurrent turbinate surgery. Only Dinesh Kumar et al. reported that septoplasty with concurrent turbinate surgery resulted in significantly greater improvement in symptoms than septoplasty alone, but the basis for this conclusion was unclear due to inconsistent reporting of results within this study ${ }^{(29)}$.

\section{Objective outcome measures}

Objective outcomes were reported in seven studies. Four types of objective outcome measures were described, i.e., active anterior rhinomanometry, acoustic rhinometry, CT measurements of turbinate thickness, and measurements of inspiratory air temperature and absolute humidity. Each objective outcome was used in one study apart from AR, which was applied in four studies. Three reports indicated that AAR or AR had been performed after decongestion of nasal mucosa; Devseren et al. and Ye et al. did not specify whether this was the case ${ }^{(28,30)}$. Most studies reported significant improvement in objective outcomes after treatment ${ }^{(28,30,31,33,34)}$. Five out of seven studies found no additional benefit of concurrent turbinate surgery $(25,28,30,32,33)$.

\section{Complications}

Only three studies reported on complications ${ }^{(30,31,34)}$. The most frequent complications were development of nasal septal perforation and nasal adhesions or synechiae, with a reported total of seven and six affected patients out of 233, respectively. Other complications were nasal hematoma and secondary hemorrhage, each of which occurred in one out of 233 patients ${ }^{(34)}$. In all three studies, complications were more frequent after septoplasty with concurrent turbinate surgery than after septoplasty alone.

\section{Discussion}

\section{Summary of evidence}

This systematic review includes five randomised controlled trials and six controlled before-and-after studies comparing the effectiveness of septoplasty with concurrent turbinate surgery to septoplasty alone for nasal obstruction due to a deviated nasal septum in adults. Included studies demonstrated substantial heterogeneity in study population, outcomes measured, and time points of outcome assessment. Risk of bias was considered high in most reports. Therefore we decided not to perform meta-analyses, but instead present effect estimates of individual studies. Subjective and objective outcomes generally appeared to have improved after treatment. However, the additional benefit of turbinate surgery was not evident. Moreover, subjective benefit was not always accompanied by objective improvement, and vice versa. Complications appeared to be rare and were re- 


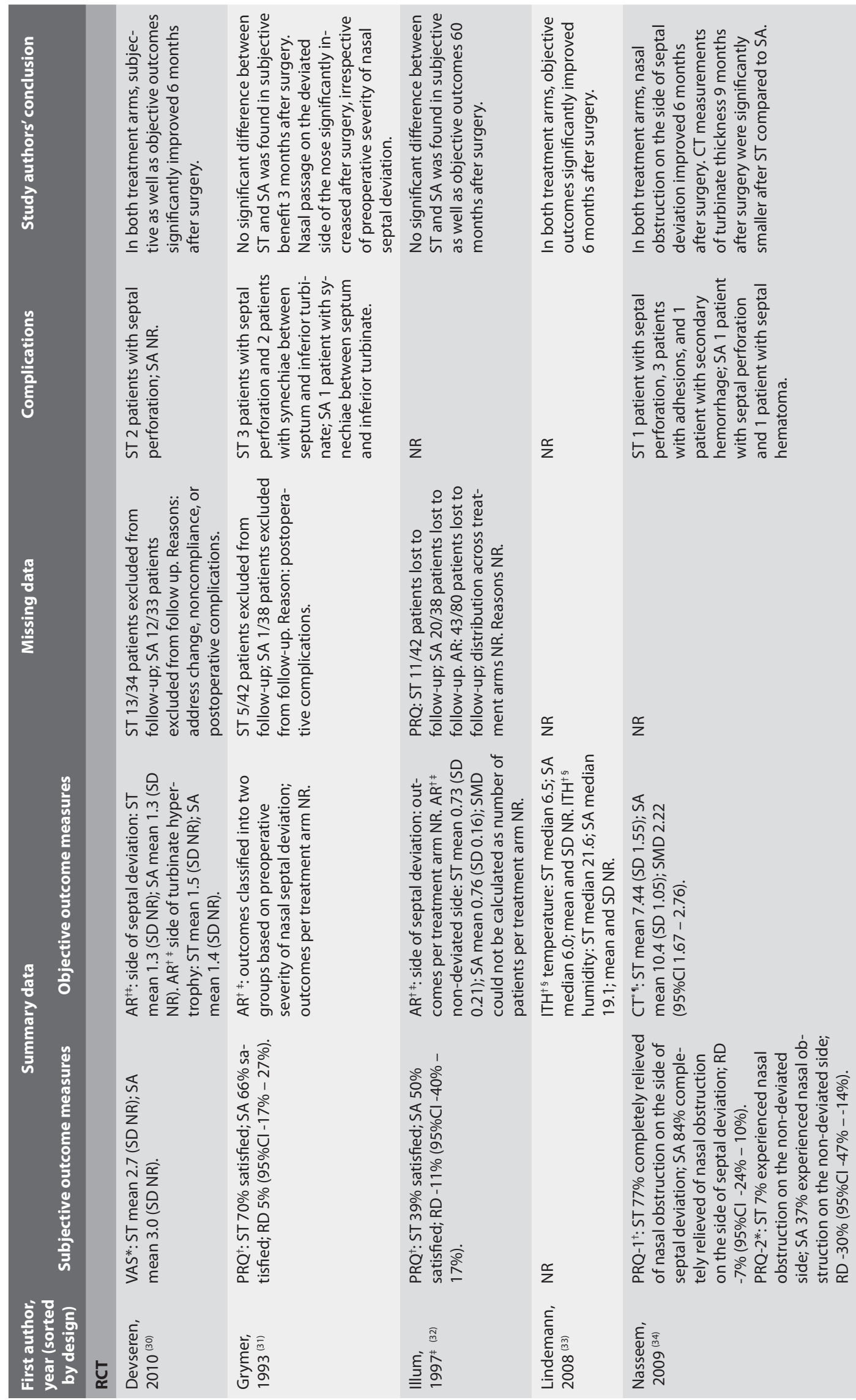




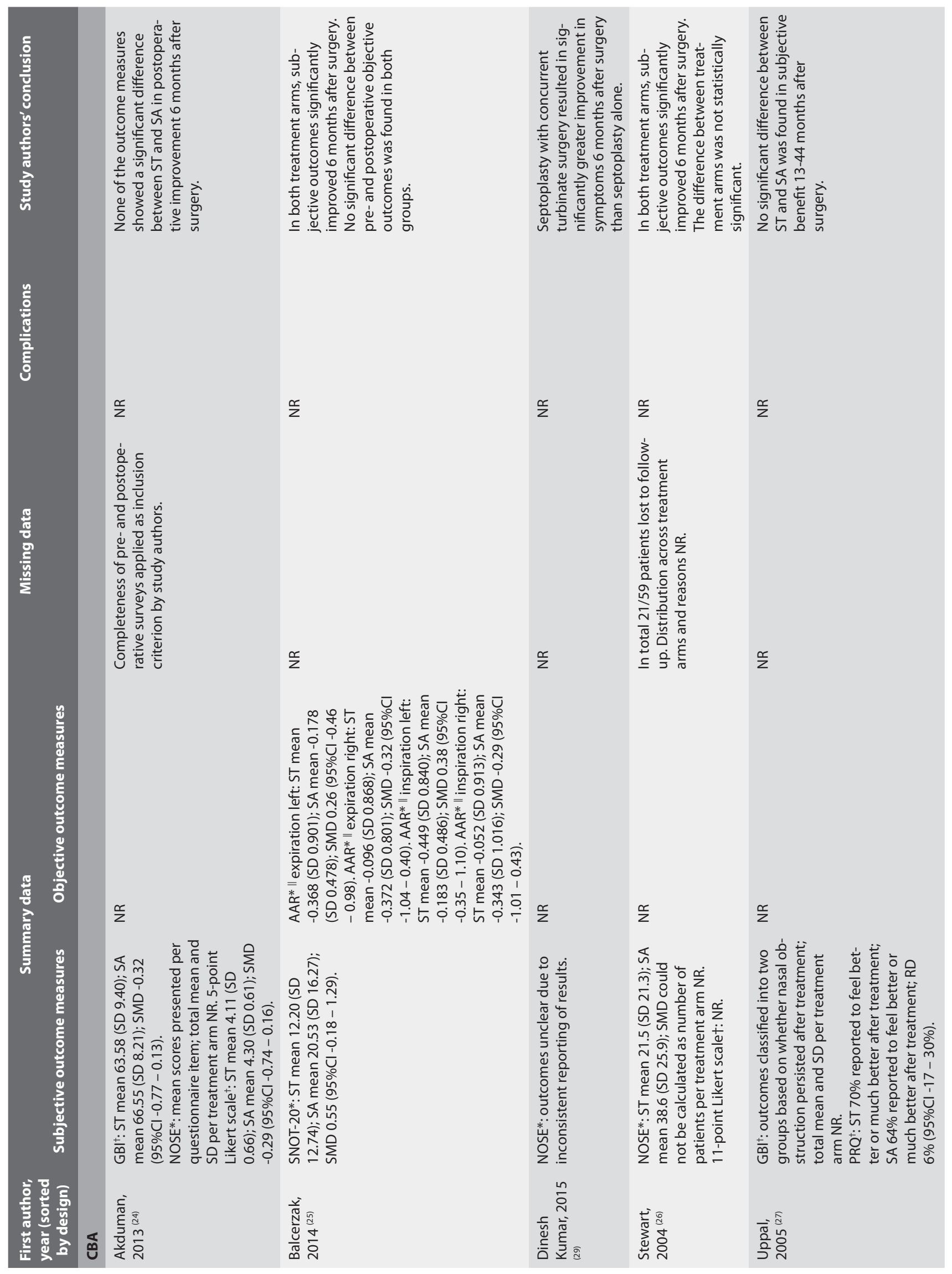




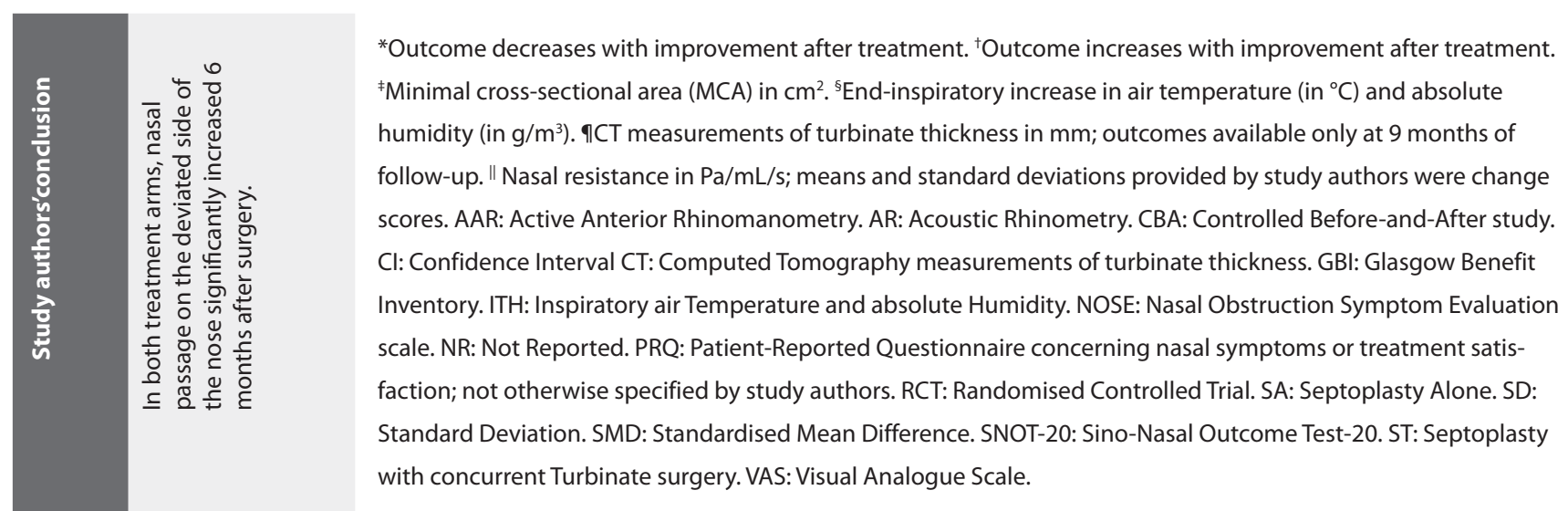

$\stackrel{\frac{\alpha}{z}}{2}$

$\stackrel{\frac{c}{z}}{2}$

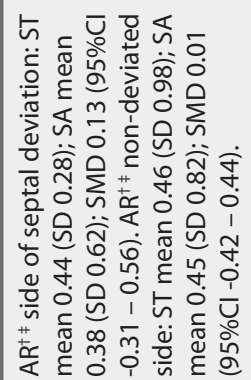

$\stackrel{\infty}{2}$

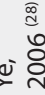

ported in only three studies. Most complications occurred after septoplasty with concurrent turbinate surgery.

\section{Strengths and limitations}

The major strength of our systematic review is that we are the first to provide an extensive evaluation of current evidence for the effectiveness of septoplasty (with or without concurrent turbinate surgery) as well as for the effectiveness of septoplasty with concurrent turbinate surgery compared to septoplasty alone, both in terms of subjective and objective outcome measures. Considering its annual performance rate combined with the existing lack of management consensus, evidence for the effectiveness of septoplasty is of high relevance to many healthcare providers, patients, and policy makers. This systematic review has been performed with strict adherence to the registered review protocol and following PRISMA guidelines ${ }^{(23)}$.

However, several limitations should be addressed as well. First, a non-surgical control group was lacking in all eligible studies. Since improvement in complaints could also be induced by other factors such as natural history, beneficial effects of surgery may be overestimated. Some study authors considered septoplasty the only possible treatment for a deviated nasal septum ${ }^{(24,26)}$. Yet the primary aim of septoplasty is reducing symptoms of nasal obstruction, rather than merely straightening the septum. For this purpose, non-surgical management is an equally suited alternative under conditions of clinical equipoise ${ }^{(35)}$. Second, follow-up of most included studies was relatively short. This may provide a distorted view on the effectiveness of septoplasty, as long-term results tend to be less favourable than short-term outcomes. Illum et al. found that only 39 to 50 percent of patients remained satisfied 5 years after treatment ${ }^{(32)}$. An uncontrolled study by Jessen et al. showed that the proportion of patients relieved of nasal obstruction dropped from 51 percent at 9 months of follow-up to 26 percent after 9 years (36). To assess durability of symptom improvement, studies with longer follow-up are needed.

Third, we used Cochrane's risk of bias tool instead of the 
Newcastle-Ottawa Scale (NOS), which may seem more appropriate for non-randomised studies ${ }^{(37)}$. However, Cochrane's risk of bias tool is tailored to our type of systematic review, addressing the effectiveness of interventions ${ }^{(22)}$. Moreover, the validity and reliability of the NOS have been questioned, and its evaluation is still in progress ${ }^{(37-39)}$. Cochrane's tool has been developed for RCTs, but the dimensions of bias to be assessed are accordingly applicable to non-randomised studies ${ }^{(22)}$.

Fourth, systematic reviews of non-randomised studies entail particular difficulties, especially when meta-analyses are involved. Supplementing evidence from RCTs with non-randomised studies bears the risk of changing an imprecise but unbiased estimate into a precise but biased one ${ }^{(22)}$. Nonetheless, including non-randomised studies may allow a systematic review to address interventions not studied in RCTs, and examine the case for undertaking future trials ${ }^{(22,40)}$. Recent years have seen an increase in literature dedicated to methodological issues surrounding systematic reviews of non-randomised studies ${ }^{(41,42)}$. When randomised and non-randomised evidence are available, including both in the same systematic review but synthesising their results separately may be considered the preferred approach ${ }^{(40)}$.

\section{Clinical implications}

Uncertainty about the effectiveness of septoplasty has been a long-time concern in ENT-practice. Accordingly, it was the clinical experience of ENT-surgeons that prompted a call for further research. In 2010, the professional association of UK otorhinolaryngologists noted that some hospital administrations were suggesting to abolish or severely restrict septoplasty, because of doubts about its benefits ${ }^{(12)}$. The Dutch ENT society highly prioritised research on this topic, considering it one of the most important knowledge gaps in otorhinolaryngology ${ }^{(13)}$. Our extensive literature search shows that the evidence gap persists. We were unable to include studies comparing septoplasty to non-surgical management, and studies comparing septoplasty with concurrent turbinate surgery to septoplasty alone were limited in number and methodologically flawed. As a result, evidence-based conclusions regarding the effectiveness of septoplasty still cannot be drawn. To assess the effectiveness of interventions, a pragmatic randomised controlled trial remains the design of choice ${ }^{(43)}$. After years of routinely performing sep- tal surgery, it may seem challenging to initiate an RCT comparing septoplasty to non-surgical management. Two such trials, however, are currently underway ${ }^{(2,44)}$. Together their results will help to determine which patients can benefit from septoplasty to a greater or lesser extent.

\section{Conclusion}

Although septoplasty is routinely applied in clinical practice, the current body of evidence does not support firm conclusions on its effectiveness. No studies were found comparing septoplasty to non-surgical management. The limited number of studies comparing septoplasty with concurrent turbinate surgery to septoplasty alone generally showed postoperative improvement, but their results must be interpreted with caution due to methodological flaws.

\section{Acknowledgements}

The review authors would like to thank all corresponding study authors who provided us with additional information on their studies; Dr. Maggie Westby, Methods Adviser of Cochrane Collaboration's Ear, Nose, and Throat Disorders Group, who gave us feedback on an earlier draft of the review protocol; Ms. Rikie Deurenberg - Vos, information specialist, who reviewed our electronic search strategy; and Ms. Carine Hendriks, trial coordinator, who supported $\mathrm{MvE}, \mathrm{MR}$, and $\mathrm{NvH}$ in their research endeavours. MvE, MR, and $\mathrm{NvH}$ performed this systematic review alongside their randomised controlled trial on the effectiveness of septoplasty. This trial is funded by ZonMw (The Netherlands Organization for Health Research and Development) and Radboud university medical center Nijmegen.

\section{Authorship contribution}

$\mathrm{MvE}, \mathrm{MR}$, and $\mathrm{NvH}$ conceived the systematic review and initiated the review protocol. AT and MvE performed the systematic literature search. AT retrieved applicable reports. $\mathrm{MvE}, \mathrm{MR}$, and $\mathrm{NvH}$ selected studies, assessed risk of bias, and extracted data. MvE and MR performed data analyses. All review authors drafted this manuscript and approved the final version.

\section{Conflict of interest}

The authors declare that they do not have any competing interests.

\section{References}

1. Manoukian PD, Wyatt JR, Leopold DA, Bass EB. Recent trends in utilization of procedures in otolaryngology-head and neck surgery. Laryngoscope 1997; 107(4): 472-7.

2. van Egmond MM, Rovers MM, Hendriks $C T$ van Heerbeek N. Effectiveness of septoplasty versus non-surgical management for nasal obstruction due to a deviated nasal septum in adults: study protocol for a randomized controlled trial. Trials 2015; 16: 500.

3. Statistische Bundesamt. Operationen und Prozeduren in Krankenhäusern. www.gbebund.de 2015.

4. Dutch Hospital Data. Utrecht, the Netherlands 2014

5. Hospital Episode Statistics, NHS Digital. Admitted Patient Care - England, 2014-
15: Procedures and interventions. Health \& Social Care Information Centre. Leeds, England 2015.

6. Bhattacharyya N. Ambulatory sinus and nasal surgery in the United States: demographics and perioperative outcomes. Laryngoscope 2010; 120(3): 635-8.

7. Jessen M, Malm L. Definition, prevalence and development of nasal obstruction. 
Allergy 1997; 52(40 Suppl): 3-6.

8. Akoglu E, Karazincir S, Balci A, Okuyucu S, Sumbas H, Dagli AS. Evaluation of the turbinate hypertrophy by computed tomography in patients with deviated nasal septum. Otolaryngol Head Neck Surg 2007; 136(3): 380-4.

9. Aaronson NL, Vining EM. Correction of the deviated septum: from ancient Egypt to the endoscopic era. Int Forum Allergy Rhinol. 2014;4(11):931-6.

10. Roblin DG, Eccles R. What, if any, is the value of septal surgery? Clin Otolaryngol 2002; 27(2): 77-80.

11. Han JK, Stringer SP, Rosenfeld RM, et al Clinical Consensus Statement: Septoplasty with or without Inferior Turbinate Reduction. Otolaryngol Head Neck Surg 2015; 153(5): 708-20.

12. ENT UK: the British Academic Conference in Otolaryngology (BACO) and the British Association of Otorhinolaryngology - Head and Neck Surgery (BAO-HNS). Nasal Septal Surgery: ENTUK position paper 2010.

13. Dutch ENT Society. [The ENT Science Agenda] 2013.

14. van Egmond MM, van Heerbeek N, Ter Haar EL, Rovers MM. Clinimetric properties of the Glasgow Health Status Inventory, Glasgow Benefit Inventory, Peak Nasal Inspiratory Flow, and 4-Phase Rhinomanometry in adults with nasal obstruction. Rhinology 2017: 55(2): 126-134.

15. Robinson K, Gatehouse S, Browning GG. Measuring patient benefit from otorhinolaryngological surgery and therapy. Ann Otol Rhinol Laryngol 1996; 105(6): 415-22.

16. Stewart MG, Witsell DL, Smith TL, Weaver EM, Yueh B, Hannley MT. Development and validation of the Nasal Obstruction Symptom Evaluation (NOSE) scale. Otolaryngol Head Neck Surg 2004; 130(2): 157-63.

17. Piccirillo JF, Merritt MG, Jr., Richards ML. Psychometric and clinimetric validity of the 20-Item Sino-Nasal Outcome Test (SNOT20). Otolaryngol Head Neck Surg 2002; 126(1): 41-7.

18. Casey KP, Borojeni AA, Koenig LJ, Rhee JS, Garcia GJ. Correlation between Subjective Nasal Patency and Intranasal Airflow Distribution. Otolaryngol Head Neck Surg 2017; 156(4): 741-50.

19. Jeong JI, Hong SD, Kim SJ, Dhong HJ, Chung SK, Kim HY. Temporal differences in improvement of nasal obstruction between primary and revision septoplasty. Am J Rhinol Allergy 2016; 30(4): 134-8.

20. Ottaviano G, Fokkens WJ. Measurements of nasal airflow and patency: a critical review with emphasis on the use of peak nasal inspiratory flow in daily practice. Allergy. 2016; 71(2): 162-74

21. Clement PA, Halewyck S, Gordts F, Michel O. Critical evaluation of different objective techniques of nasal airway assessment: a clinical review. Eur Arch Otorhinolaryngol 2014: 271(10): 2617-25.

22. Higgins JPT, Green S. Cochrane Handbook for Systematic Reviews of Interventions Version 5.1.0. www.handbook.cochrane.org 2011

23. Moher D, Liberati A, Tetzlaff J, Altman DG, Group P. Preferred reporting items for systematic reviews and meta-analyses: the PRISMA statement. PLoS Med 2009; 6(7) e1000097.

24. Akduman D, Yanilmaz M, Haksever M, Doner F, Sayar Z. Patients' evaluation for the surgical management of nasal obstruction. Rhinology 2013; 51(4): 361-7.

25. Balcerzak J, Lukawska I, Grzanka A Niemczyk K. [Comparative analysis of the treatment results of the nasal obstruction using septoplasty and septokonchoplasty]. Otolaryngol Pol 2014; 68(3): 129-34

26. Stewart MG, Smith TL, Weaver EM, Witsell DL, Yueh B, Hannley MT, et al. Outcomes after nasal septoplasty: results from the Nasal Obstruction Septoplasty Effectiveness (NOSE) study. Otolaryngol Head Neck Surg 2004; 130(3): 283-90.

27. Uppal S, Mistry H, Nadig S, Back G, Coatesworth A. Evaluation of patient benefit from nasal septal surgery for nasal obstruction. Auris Nasus Larynx 2005; 32(2): 129-37

28. Ye QY, Liao J, Wang $H$, Sun A. "Nasal modification" for nasal septum deviation: Assessment of clinical outcomes. Acad J Second Mil Med Univ 2006; 27(8): 905-6.

29. Dinesh Kumar R, Rajashekar M. Comparative Study of Improvement of Nasal Symptoms Following Septoplasty with Partial Inferior Turbinectomy Versus Septoplasty Alone in Adults by NOSE Scale: A Prospective Study. Indian J Otolaryngol Head Neck Surg 2016; 68(3): 275-84

30. Devseren NO, Ecevit MC, Erdag TK, Ceryan K. A randomized clinical study: outcome of submucous resection of compensatory inferior turbinate during septoplasty. Rhinology 2011; 49(1): 53-7.

31. Grymer LF, Illum P, Hilberg O. Septoplasty and compensatory inferior turbinate hypertrophy: a randomized study evaluated by acoustic rhinometry. J Laryngol Otol 1993; 107(5): 413-7.

32. Illum P. Septoplasty and compensatory inferior turbinate hypertrophy: long-term results after randomized turbinoplasty. Eur Arch Otorhinolaryngol 1997; 254 Suppl 1 S89-92

33. Lindemann J, Keck T, Leiacker R, Dzida R Wiesmiller K. Early influence of bilateral turbinoplasty combined with septoplasty on intranasal air conditioning. Am J Rhino 2008; 22(5): 542-5.

34. Nasseem N.T. AFAF, Mohamad A.S., Ashou B.M., Wahba M.H. Turbinate reduction during septoplasty: to do it or not? Clinical and radiological study. Med J Cairo Univ 2009; 77(1): 391-5.

35. Sipila J, Suonpaa J. A prospective study using rhinomanometry and patient clinical satisfaction to determine if objective measurements of nasal airway resistance can improve the quality of septoplasty. Eur
Arch Otorhinolaryngol 1997; 254(8): 387-90.

36. Jessen M, Ivarsson A, Malm L. Nasal airway resistance and symptoms after functional septoplasty: comparison of findings at 9 months and 9 years. Clin Otolaryngol 1989; 14(3): 231-4

37. Wells GA, Shea B, O'Connell D, et al. The Newcastle-Ottawa Scale (NOS) for assessing the quality of nonrandomised studies in meta-analyses. http://www.ohri.ca/ programs/clinical_epidemiology/oxford.asp 2008

38. Stang A. Critical evaluation of the Newcastle-Ottawa scale for the assessment of the quality of nonrandomized studies in meta-analyses. Eur J Epidemiol. 2010; 25(9): 603-5.

39. Hartling L, Milne A, Hamm MP, et al. Testing the Newcastle Ottawa Scale showed low reliability between individual reviewers. Clin Epidemiol. 2013; 66(9): 982-93.

40. Valentine JC, Thompson SG. Issues relating to confounding and meta-analysis when including non-randomized studies in systematic reviews on the effects of interventions. Res Synth Methods 2013; 4(1): 26-35.

41. Wells GA, Shea B, Higgins JP, Sterne J, Tugwell P, Reeves BC. Checklists of methodological issues for review authors to consider when including non-randomized studies in systematic reviews. Res Synth Methods 2013; 4(1): 63-77.

42. Schunemann HJ, Tugwell P, Reeves BC, et al. Non-randomized studies as a source of complementary, sequential or replacement evidence for randomized controlled trials in systematic reviews on the effects of interventions. Res Synth Methods. 2013; 4(1): 49-62.

43. Zwarenstein M, Treweek S, Gagnier JJ, et al. Improving the reporting of pragmatic trials: an extension of the CONSORT statement BMJ. 2008; 337: a2390.

44. Carrie S. Nasal airway obstruction study NAIROS. https://doi.org/10.1186/ ISRCTN16168569. Date last accessed: 15 February 2018.

M.M.H.T. van Egmond, MD, MSc Radboud university medical center Department of Otorhinolaryngology route 377

P.O. Box 9101 6500 HB Nijmegen the Netherlands

$\mathrm{T}:+31-24-3614178$

$\mathrm{F}:+31-24-3540251$

E: Machteld.vanEgmond@radboud umc.nl 